\title{
Revisión sistemática de procedimientos quirúrgicos usados para tratar patología aórtica en México
}

\author{
Luis Barragán-Galindo, Alejandra Soto-Pérez, Javier E. Anaya-Ayala, Ramón García-Alva, César Cuen- \\ Ojeda y Carlos A. Hinojosa \\ Secretaría de Salud, Instituto Nacional de Ciencias Médicas y Nutrición "Salvador Zubirán", Dirección de Cirugía, Ciudad de México, México
}

\begin{abstract}
Resumen
Introducción: En México no se cuentan con estadísticas adecuadas acerca de la enfermedad aórtica, que puede ser tratada mediante manejo médico o cirugía abierta o endovascular. Objetivo: Identificar la literatura en bases de datos electrónicas relativa al manejo invasivo de la patología aórtica en México. Método: Se realizó búsqueda sistemática y revisión narrativa de la literatura disponible en diferentes bases de datos electrónicas: PubMed, Imbiomed, Clinical Key, Bibliat, Scielo, Sage Journals, Sciencedirect y con el motor de búsqueda de Google Académico. Resultados: Se encontraron 90 artículos, de los cuales 50 reunían los criterios de inclusión. Fueron seleccionados estudios observacionales y reportes de casos, con énfasis en los datos demográficos de los pacientes, resultados clínicos y la supervivencia posoperatoria a 30 días. Conclusión: Se reporta elevada tasa de complicaciones de las enfermedades aórticas debido al infradiagnóstico de estas, que deriva en manejo más complejo y pronóstico desfavorable. La creación de un registro nacional de patología aórtica es crucial para la estandarización en el abordaje y la optimación de los resultados.
\end{abstract}

PALABRAS CLAVE: México. Aorta. Aneurismas. Coercion. Trauma. Disección aórtica.

\begin{abstract}
Introduction: In Mexico, there are no appropriate statistical data on aortic disease, which can be treated with medical management or open or endovascular surgical approach. Objective: To carry out a systematic search and review of the literature in electronic databases with regard to invasive management of aortic pathology in Mexico. Method: A systematic search and narrative review of available literature was carried out using different electronic databases: PubMed, Imbiomed, Clinical Key, Bibliat, Scielo, Sage Journals and Sciencedirect, as well as with the Google Scholar search engine. Results: Ninety articles were found, out of which only 53 met the inclusion criteria. Observational studies and case reports were selected, emphasizing on patient demographics, clinical results, and 30-day postoperative survival. Conclusion: An elevated rate of complications is reported for aortic pathologies due to underdiagnosis, which results in management being more complicated and prognosis unfavorable. The creation of a national aortic disease registry is crucial to standardization in the approach and optimization of results.
\end{abstract}

KEY WORDS: Mexico. Aorta. Aneurysms. Coarctation. Trauma. Aortic dissection.

Fecha de recepción: 24-05-2018

Fecha de aceptación: 24-11-2018

DOI:10.24875/GMM.18004420
Gac Med Mex. 2019;155:136-142

Disponible en PubMed www.gacetamedicademexico.com 


\section{Introducción}

Las enfermedades aórticas forman parte del amplio espectro de patologías arteriales como los aneurismas aórticos, el pseudoaneurisma, la ruptura aórtica, afecciones inflamatorias, enfermedades genéticas y síndromes aórticos agudos.

En México, actualmente no se cuentan con estadísticas adecuadas acerca de la enfermedad aórtica debido a que es infradiagnosticada y con frecuencia la causa de muerte es atribuida a otras patologías. El diagnóstico oportuno va en aumento gracias a los nuevos métodos diagnósticos de mínima invasión y a los nuevos programas de búsqueda intencional. ${ }^{1}$

Para el tratamiento se puede optar por manejo médico o abordaje quirúrgico, abierto o endovascular. La cirugía abierta ha sido el estándar terapéutico de diversas patologías aórticas de alta morbimortalidad. Aunque los procedimientos de mínima invasión paulatinamente han desplazado a la cirugía abierta, esta última sigue siendo necesaria en algunas patologías aórticas y para el manejo de complicaciones que puedan surgir del tratamiento endovascular.

El aneurisma aórtico se define como una dilatación focal de la aorta que excede $50 \%$ el diámetro normal de esta y resulta del debilitamiento progresivo de sus paredes; si involucra a las tres capas murales (íntima, media y adventicia) se considera aneurisma verdadero; los pseudoaneurismas se forman como resultado de una lesión en la pared aórtica donde el flujo de sangre extraluminal permanece en el tejido circundante. Su localización más frecuente $(90 \%)$ es en la aorta abdominal, específicamente en su porción infrarrenal. Entre los factores de riesgo más importantes se encuentran el sexo masculino, edad mayor a 65 años, tabaquismo, antecedentes heredofamiliares, hipertensión y dislipidemias. La complicación más grave es la rotura del aneurisma, asociada con una tasa de mortalidad de $80 \%$.

Por otra parte, la disección se define como la separación de las capas de la pared aórtica. Desgarros en la íntima del vaso pueden provocar que la disección se expanda, tanto proximal como distalmente, debido a que la sangre ocupa el espacio entre la capa íntima y la media. A pesar de los avances tecnológicos en métodos diagnósticos y terapéuticos, la mortalidad por esta patología sigue siendo alta. Las disecciones aórticas se deben tanto a factores genéticos como ambientales y su prevalencia es mayor en pacientes con desórdenes del tejido conjuntivo, hipertensión arterial sistémica, estenosis aórtica congénita o válvula aórtica bicúspide.

La coartación aórtica se caracteriza por engrosamiento anormal de la capa media e hiperplasia de la íntima. Representa hasta $7 \%$ de las cardiopatías congénitas, con una prevalencia de 0.2 por cada 1000 nacidos vivos y con una relación hombre-mujer de 2:1. Es común que afecte la pared posterior al comienzo de la arteria subclavia, principalmente al arco aórtico distal y la aparición de la aorta descendente. De igual manera se acompaña de anomalías cardiacas, como válvula aórtica bicúspide, conducto arterioso permeable o defectos del tabique ventricular y es una de las anomalías que se encuentran comúnmente en síndromes genéticos, como el de Turner. ${ }^{2}$

Por último, el trauma vascular se ha convertido en un problema crítico de salud. Se puede englobar principalmente en accidentes de tránsito o lesiones generadas por violencia, las cuales representan de las primeras causas de muerte en población joven. ${ }^{3} \mathrm{La}$ lesión aórtica por trauma contuso y desaceleración es la segunda causa más frecuente de muerte por accidentes de tráfico severos. La ruptura aórtica puede ocurrir a cualquier nivel de la aorta torácica, si bien es menos frecuente en la aorta abdominal. ${ }^{4}$ El diagnóstico y tratamiento oportunos son primordiales para la supervivencia. El tratamiento endovascular es una excelente alternativa debido a que disminuye la morbilidad, la mortalidad, el tiempo quirúrgico y la estancia intrahospitalaria. ${ }^{5}$

\section{Método}

Se realizó una búsqueda sistemática en las bases de datos electrónicas PubMed (www.ncbi.nlm.nih.gov/ pubmed), Imbiomed (www.imbiomed.com.mx), Clinical Key (www.clinicalkey.com), Bibliat (www.bibliat. unam.mx/es/), Scielo (www.scielo.org), Sage Journals (www.journals.sagepub.com), Science direct (www. sciencedirect.com) y con el motor de búsqueda de Google Académico. Como palabras clave se utilizaron los términos "México" y "aorta".

En la Figura 1 se esquematiza el proceso de la revisión sistemática de la literatura. Se seleccionaron únicamente los artículos realizados en México y se excluyeron los relacionados con valvulopatía aórtica.

\section{Resultados}

En los 50 artículos seleccionados se identificaron 1383 pacientes. Los trabajos fueron agrupados de 


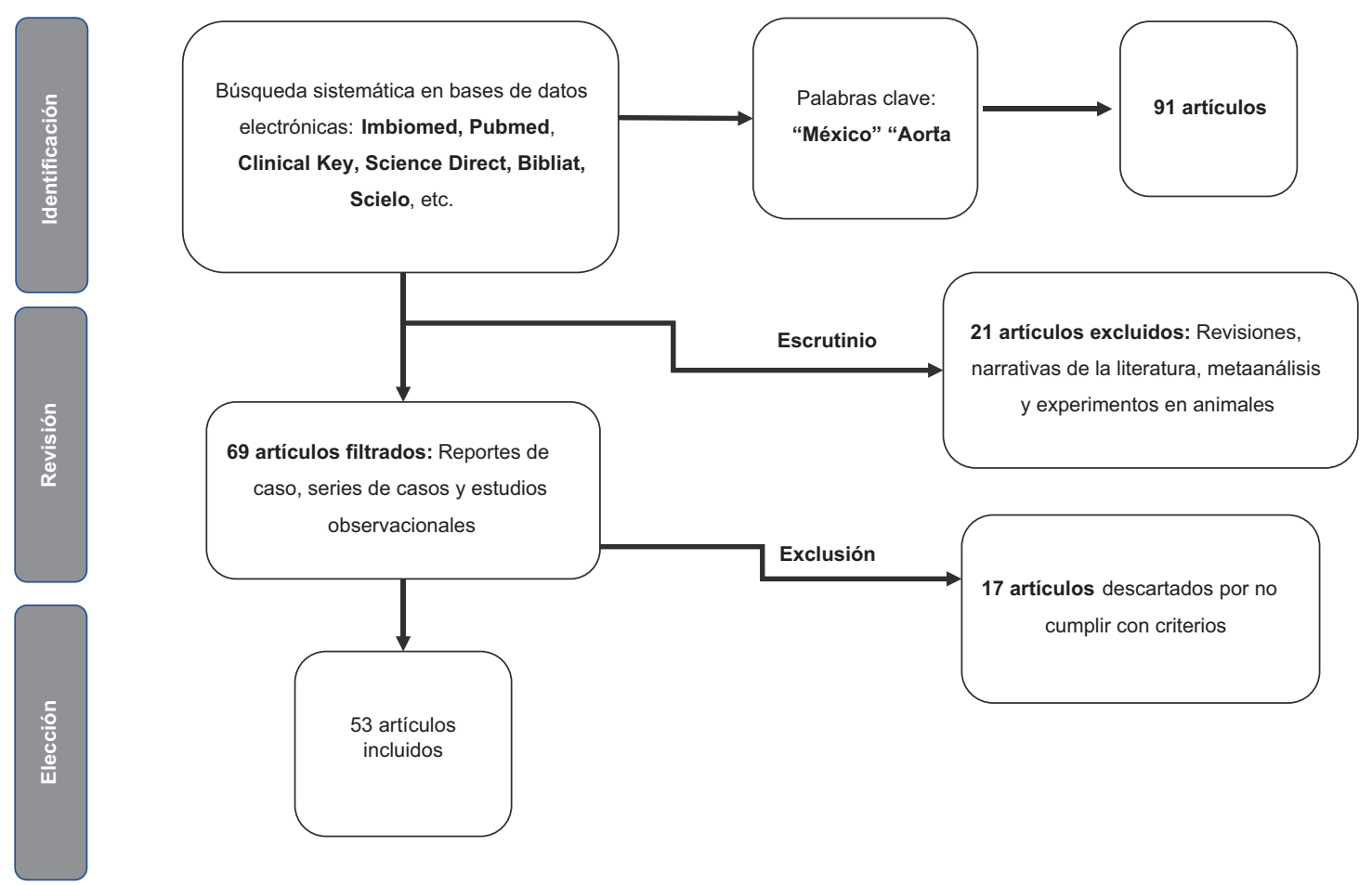

Figura 1. Diagrama de flujo. Búsqueda sistemática de la literatura acerca de patología aórtica.

acuerdo con el tipo de patología aórtica y el análisis descriptivo de la estadística se clasificó en dos grupos: estudios observacionales y reportes de caso. Se agregó el apartado "otras patologías aórticas", debido a que no se encontraron suficientes estudios de algunas patologías para realizar el análisis individual.

\section{Aneurisma aórtico}

Se revisaron 13 artículos observacionales en los cuales se incluyeron pacientes con aneurisma de la aorta torácica y abdominal. Se realizó la estadística descriptiva determinando las desviaciones estándar y las medias. Se ponderaron los resultados de acuerdo con el número de pacientes por artículo (Tabla 1). El total de los pacientes incluidos en los artículos fue de 418 , con una media de edad de 64 años, $78 \%$ de los pacientes fue del sexo masculino y $80 \%$ se manejó con cirugía abierta; la supervivencia a 30 días fue de $78 \%{ }^{6-18}$

También se analizaron reportes de caso, divididos conforme a la localización del aneurisma (Tabla 2). ${ }^{19-29}$ Se encontraron reportes de cinco pacientes con aneurismas de la aorta abdominal, en los cuales la edad media fue 70 años y $60 \%$ fueron pacientes masculinos; $60 \%$ fue tratado de forma endovascular y electiva, con una supervivencia a 30 días de $80 \%$.
Se encontraron reportes de caso de seis pacientes con aneurismas de la aorta torácica, la edad media fue de 55 años y $83 \%$ era del sexo masculino; $72 \%$ fue tratado de forma endovascular y electiva, resultando en una supervivencia a 30 días de $83 \%$.

En la Figura 2A se puede apreciar la reconstrucción tridimensional de una angiotomografía de un paciente con aneurisma de la aorta infrarrenal.

\section{Coartación aórtica}

Se encontraron ocho publicaciones observacionales correspondientes a coartación aórtica (Tabla 1)..$^{30-37} \mathrm{Se}$ cuantificaron 761 pacientes, cuya media de edad fue de 13 años y $70 \%$ correspondió al sexo masculino. En cuanto al tratamiento, $58 \%$ fue intervenido en forma endovascular. Específicamente es esta enfermedad se evaluó el gradiente medio transcoartación, tanto previo como posterior a la intervención. El gradiente medio de la presión anterior al tratamiento fue de $27 \mathrm{~mm} \mathrm{Hg}$ y el posterior, de $6 \mathrm{~mm} \mathrm{Hg}$. La media de la supervivencia a 30 días fue de $95 \%$.

Para la estadística descriptiva de los reportes de caso (Tabla 2) 28-43 $^{38}$ usaron las mismas variables. Se encontraron seis reportes, en los cuales la edad promedio fue de 17 años y $50 \%$ de los pacientes fue del sexo masculino. La mitad recibió tratamiento 
Tabla 1. Estudios observacionales sobre el tratamiento invasivo de diferentes patologías aórticas

\begin{tabular}{|c|c|c|c|c|c|c|}
\hline $\begin{array}{l}\text { Número de } \\
\text { artículo }\end{array}$ & Autor & $\begin{array}{l}\text { Pacientes } \\
\text { (n) }\end{array}$ & $\begin{array}{l}\text { Hombres } \\
\text { (\%) }\end{array}$ & $\begin{array}{l}\text { Media de edad } \\
(\%)\end{array}$ & $\begin{array}{c}\text { Tratamiento abierto } \\
\text { (\%) }\end{array}$ & $\begin{array}{c}\text { Supervivencia a } 30 \text { días } \\
(\%)\end{array}$ \\
\hline \multicolumn{7}{|c|}{ Aneurisma aórtico } \\
\hline 1 & Rojas $(1996)^{6}$ & 31 & 90.30 & 64.7 & 100 & 76.90 \\
\hline 2 & Mendoza $(2001)^{7}$ & 20 & 75 & 67.8 & 100 & 100 \\
\hline 3 & Rojas $(2002)^{8}$ & 75 & 88 & 68.61 & 100 & 66 \\
\hline 4 & Ramírez $(2004)^{9}$ & 4 & 100 & 62.5 & 100 & 50 \\
\hline 5 & González (1996)10 & 20 & 20 & 71 & 100 & 10 \\
\hline 6 & Álvarez (2002) ${ }^{11}$ & 101 & 74.20 & 69.1 & 100 & 72 \\
\hline 7 & Marquina (2008) $)^{12}$ & 54 & 75.90 & 71.9 & 35.18 & 94.70 \\
\hline 8 & Serrano $(2003)^{13}$ & 6 & 33.30 & 41 & 100 & 100 \\
\hline 9 & Rodríguez (2011) & 12 & 83.30 & 57.8 & 0 & 100 \\
\hline 10 & Fink $(2002)^{15}$ & 31 & 90.30 & 69.1 & 0 & 96.80 \\
\hline 11 & Ramírez $(2003)^{16}$ & 39 & 82.05 & 47 & 100 & 89.80 \\
\hline 12 & Careaga $(2009)^{17}$ & 2 & 100 & 11 & 100 & 100 \\
\hline 13 & Galicia $(2010)^{18}$ & 23 & 83 & 46 & 83 & 87 \\
\hline Totales & & 418 & $77.99 \%$ & $64.6 \pm 17.4$ & 80.4 & 78.05 \\
\hline \multicolumn{7}{|c|}{ Coartación aórtica } \\
\hline 1 & González (2006) 30 & 7 & 85.70 & 24 & 0 & 100 \\
\hline 2 & Uruchurtu $(1999)^{31}$ & 6 & 83.30 & 27.1 & 0 & 100 \\
\hline 3 & Munayer (2002) $)^{32}$ & 272 & 73.50 & 8.1 & 0 & 98.50 \\
\hline 4 & Fink $(2002)^{33}$ & 4 & 100 & 23.1 & 0 & 100 \\
\hline 5 & Hernández $(2003)^{34}$ & 58 & 75.80 & 7 & 70 & 94.93 \\
\hline 6 & Márquez (2015)35 & 166 & 71 & 18 & 36.70 & 100 \\
\hline 7 & Zabal $(2003)^{36}$ & 32 & 63 & 24.00 & 0 & 100 \\
\hline 8 & Cervantes $(2006)^{37}$ & 216 & 61 & 13 & 100 & 86.20 \\
\hline Totales & & 761 & 69.4 & $12.9 \pm 7.9$ & 41.72 & 95.16 \\
\hline \multicolumn{7}{|c|}{ Otras patologías aórticas } \\
\hline & Espinoza $(2013)^{4}$ & 2 & 0 & 37.5 & 100 & 100 \\
\hline 1 & Guering $(2015)^{44}$ & 94 & 74 & 50.9 & 33.00 & 78.80 \\
\hline 2 & Hinojosa $(2015)^{46}$ & 2 & 0 & 37.5 & 100 & 100 \\
\hline 3 & Guering $(2013)^{45}$ & 10 & 60 & 35.1 & 100 & 88.90 \\
\hline 4 & Mijangos (2015) $)^{47}$ & 5 & 60 & 5.6 & 100 & 80 \\
\hline 5 & Herrera $(2011)^{48}$ & 3 & 100 & 28.5 & 100 & 66.60 \\
\hline 6 & Fink $(2002)^{49}$ & 2 & 100 & 34 & 0 & 100 \\
\hline
\end{tabular}

endovascular. Al igual que en los estudios observacionales, se analizó el gradiente transcoartación, cuya media pretratamiento fue de $69 \mathrm{~mm} \mathrm{Hg}$ y postratamiento, de $8 \mathrm{~mm} \mathrm{Hg}$. No se reportó ninguna defunción en los primeros 30 días posteriores al tratamiento. ${ }^{38-43}$ 
Tabla 2. Reportes de casos aislados sobre el tratamiento invasivo de diferentes patologías aórticas

\begin{tabular}{|c|c|c|c|c|c|}
\hline Patología & $\begin{array}{l}\text { Reportes } \\
\text { (n) }\end{array}$ & $\begin{array}{l}\text { Edad } \\
\text { media }\end{array}$ & $\begin{array}{l}\text { Hombres } \\
\text { (\%) }\end{array}$ & $\begin{array}{c}\text { Tratamiento abierto } \\
\text { (\%) }\end{array}$ & $\begin{array}{c}\text { Supervivencia a } 30 \text { días } \\
(\%)\end{array}$ \\
\hline Aneurismas de la aorta abdominal $\left.\right|^{19-23}$ & 5 & 70 & 60 & 40 & 80 \\
\hline Aneurismas de la aorta torácica ${ }^{24-29}$ & 6 & 55 & 83.30 & 16.70 & 83.30 \\
\hline Coartación aórtica ${ }^{38-43}$ & 6 & 17 & 50 & 50 & 100 \\
\hline Fístulas aórticas $50-52$ & 3 & 66 & 66 & 100 & 100 \\
\hline Trauma vascular $5,53,54$ & 3 & 26.33 & 0 & 33 & 100 \\
\hline Patologías aórticas congénitas ${ }^{55}$ & 1 & 4 & 0 & 100 & 100 \\
\hline
\end{tabular}

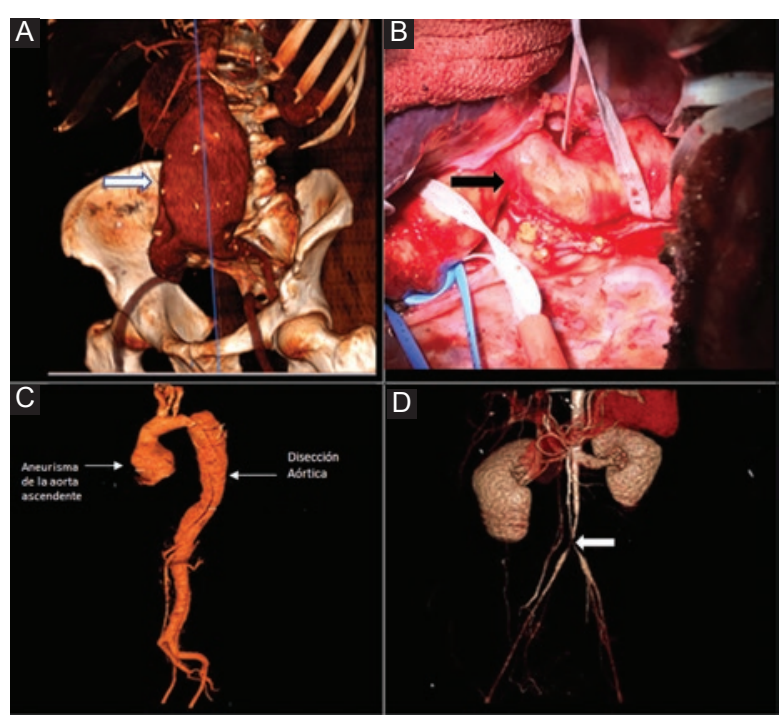

Figura 2. A) Aneurisma de la aorta abdominal en la porción infrarrenal. B) Fotografía intraoperatoria de reparación de coartación aórtica. C) Disección de la aorta descendente. D) Estenosis en la aorta abdominal en un paciente con arteritis de Takayasu (figura $2 D$ tomada de referencia 46).

En la Figura 2B se muestra el procedimiento quirúrgico abierto de una coartación aórtica.

\section{Otras patologías aórticas}

Se analizaron estudios observacionales y reportes de caso de las siguientes patologías: síndromes aórticos agudos, arteritis de Takayasu, disecciones aórticas, malformaciones congénitas, tumores con afectación vascular, trauma aórtico y fístulas. Se agruparon en la misma categoría por la poca cantidad de trabajos que se han publicado en México acerca de estos temas. La estadística descriptiva de los artículos más representativos se detalla en la Tabla 1.4,44-49

La investigación que incluyó el mayor número de pacientes fue la realizada por Guering, ${ }^{44}$ en la que se revisaron los expedientes de 94 pacientes con síndromes aórticos agudos (úlceras penetrantes, hematomas intramurales, disecciones complicadas y transecciones aórticas). Se abordó de forma endovascular a $67 \%$ de los pacientes, con supervivencia a 30 días de $79 \%$.

El único artículo identificado en torno a la disección aórtica incluyó 10 pacientes con síndrome de Marfán y disecciones tipo $B$, los cuales se intervinieron de forma abierta, con supervivencia posoperatoria a 30 días de $89 \%$ (Figura $2 \mathrm{C}$ ). ${ }^{45}$

Se encontraron dos estudios observacionales relacionados con trauma vascular que incluyeron cuatro pacientes, con una media de edad de 34 años, dos eran del sexo masculino. Todos los pacientes sufrieron trauma cerrado con afectación de la aorta torácica; a la mitad se le efectuó intervención endovascular. No hubo defunciones en los primeros 30 días posoperatorios. ${ }^{4,49}$

En el trabajo de Hinojosa se mencionan dos pacientes con arteritis de Takayasu, los cuales presentaron síndrome de aorta media como complicación, que tiene una prevalencia reportada de $0.000001 \%$ en los pacientes con dicha patología. La edad media de los pacientes fue de 37 años, ambos del sexo femenino. Fueron abordadas con cirugía abierta y la supervivencia a 30 días fue de $100 \%$. En la Figura 2D se observa una reconstrucción tridimensional de la angiotomografía de una de las pacientes mencionadas en el artículo. ${ }^{46}$

Se encontraron reportes de caso acerca de fístulas aórticas, trauma vascular y patologías vasculares congénitas (Tabla 2). ${ }^{5,50-55}$

La edad medio de los tres pacientes reportados con fístulas aórticas fue de 66 años; $66 \%$ fue del sexo masculino. Todos fueron tratados con cirugía abierta. Dos pacientes presentaron fístulas cavoaórticas, uno como consecuencia de una intervención retroperitoneal por trauma y el otro por aneurisma de la aorta abdominal. Hubo un caso de fístula aortogástrica provocado por una úlcera. La supervivencia a 30 días fue de $100 \%$. 
Se identificaron tres casos de trauma vascular; todos del sexo femenino. La edad media fue de 26 años. Dos pacientes con lesiones en la aorta abdominal fueron tratados de forma endovascular y uno con lesión en la aorta torácica fue tratado con cirugía abierta. No hubo ninguna defunción.

Por último, se informó de una niña de cuatro años que presentó anillo vascular congénito en la aorta torácica, tratada con cirugía abierta y cuya evolución posoperatoria fue favorable.

\section{Discusión}

El conocimiento y el diagnóstico oportuno de la patología aórtica en México se han incrementado en los últimos años debido a la implementación y aumento gradual de programas de escrutinio en diferentes institutos y centros de atención médica alrededor del país. No obstante, es necesario generar mayores y mejores programas con el objetivo de identificar a una mayor cantidad de individuos en riesgo, para solventar la demanda poblacional.

Aun cuando el aneurisma aórtico continúa siendo la patología aórtica más común, identificamos que en México se ha estudiado a un mayor número de pacientes con coartación aórtica; los pacientes que se incluyen en esta revisión representan $54.9 \%$ del total de pacientes, mientras que los pacientes con aneurismas aórticos, $30.15 \%$.

En relación con el manejo quirúrgico de las de las patologías aórticas analizadas, a $51.97 \%$ de los pacientes se les abordó con cirugía abierta, y al resto se manejó de manera endovascular.

Si bien en nuestro análisis solo se tomó en cuenta la supervivencia posoperatoria a los 30 días, en algunos trabajos incluidos se mencionan complicaciones posquirúrgicas. En el grupo de pacientes tratados con cirugía abierta se mencionan lesión de la vena renal, hematoma retroperitoneal, lesión renal aguda, falla respiratoria y evento vascular cerebral isquémico. Por otro lado, en el grupo con abordaje endovascular, las complicaciones más comunes fueron perforación vascular, evento vascular cerebral isquémico y trombosis en el sitio de entrada.

La tasa de mortalidad a 30 días de los pacientes sometidos a cirugía fue de $12.33 \%$. La patología que presentó la mayor tasa fue el aneurisma aórtico, con $21.8 \%$.

Es de esperar que en México, por su gran densidad poblacional y el déficit de cobertura de los sistemas para detección temprana y tratamiento oportuno, un porcentaje importante de estas patologías aórticas siga siendo subestimado e infradiagnosticado.

\section{Conclusión}

La patología aórtica continúa siendo una causa de morbimortalidad importante en México, por lo que es necesaria la formación de más especialistas con el entrenamiento quirúrgico adecuado en estas patologías y sus complicaciones.

De igual manera consideramos que la capacitación de médicos no especialistas en angiología y cirugía vascular sobre el conocimiento y diagnóstico de patologías aórticas sería de gran utilidad para detectar la enfermedad en etapas tempranas.

En cuanto al campo de investigación clínica, la patología aórtica representa un área de oportunidad en México. Consideramos que la creación de un registro nacional de enfermedad aórtica sería relevante para la optimación de los resultados clínicos. Por esta razón, podemos concluir que sería fundamental impulsar el crecimiento de la investigación, con el fin de integrar el conocimiento preciso de estas patologías y así ofrecer el manejo más oportuno para cada una.

\section{Bibliografía}

1. Martínez-Hernández $H$. Los aneurismas de la aorta torácica y su enfoque terapéutico. Arch Cardiol Mex. 2006;76:S124-S133.

2. Márquez-González H, De León Mena S, Yáñez-Gutiérrez L, López-Gallegos D, Camargo-Zetina CO, Ortiz-Vázquez IC, et al. Complicaciones tardías de tratamiento de coartación aórtica: estudio mexicano de cohorte con más de 10 años de seguimiento. Rev Mex Cardiol. 2015;26: 169-173.

3. Castañeda-Gaxiola, R. El problema del trauma vascular en México. Cir Gen. 2000;22:254-256.

4. Espinoza HJD, Nina VO, Venegas GU, Marroquín RR, Villarreal RCB, Molina E. Ruptura traumática de aorta torácica. Reporte de dos casos. Rev Latinoam Cir. 2013;3:47-50.

5. Kalach-Mussali AJ, Montes-Corrales BS, Vázquez-Minero JC. Diagnóstico oportuno de la disección de aorta por traumatismo contuso. Neumol Cir Torax. 2013;72:223-228.

6. Rojas, GA, Cervantes, J, Arcos, L, Guibovich, V, Colin, V. Aneurismas de la aorta abdominal. Cinco años de experiencia en el hospital American British Cowdray. Rev Mex Angiol. 1996;24:34-39.

7. Mendoza-Cortés A, Rodríguez-Trejo JM, Escotto-Sánchez I, Flores-PadiIla CM, Montuy-Vidal MA, Sulvarán-Aguilar A, et al. Abordaje retroperitoneal en aneurismas de aorta abdominal. Rev Mex Angiol. 2001;29:5-14.

8. Rojas GA, Cervantes J, Torrontegui Á. Análisis de resultados en cirugía de aneurismas de aorta abdominal. Experiencia de 32 años. Rev Mex Angiol. 2002;30:38-42.

9. Ramírez-González AH, Corona-Murguía A, Murillo-Estrada D, Moreno-López M, Pérez-Villagómez Y, Tinoco-Pérez S, et al. Aneurisma roto de aorta abdominal. Experiencia en Uruapan Michoacán. Rev Mex Angiol. 2004;32:126-131.

10. González LG, Sánchez C, Martínez CM. Aneurismas de aorta abdominal rotos: dos años de experiencia. Rev Mex Angiol. 1996;24:55-58.

11. Álvarez Tostado-Fernández $F$, Martínez Hernández-Magro P, Villanueva-Sáenz $E$. Colitis isquémica en pacientes sometidos a cirugía de reemplazo aórtico. Factores de riesgo. Rev Gastroenterol Mex. 2002;67:171-178.

12. Marquina ME, Rodríguez TJM, Escotto SI, Rodríguez N, Morales GLG, Espinosa APA. Complicaciones en el tratamiento de aneurisma de aorta abdominal infrarrenal del adulto mayor. Rev Mex Angiol. 2008;36:126-134.

13. Serrano CA, Martínez C, Sánchez B, García J, Vera O, Jiménez J. Experiencia quirúrgica con los aneurismas de aorta abdominal en el síndrome de Marfan. Rev Mex Angiol. 2003;31:13-17. 
14. Rodríguez-Ortega F, Hernández-Mercado MA, Reyes-Corona JA, Gómez-Ríos N, Jaymes-Nuñez A, Alegría-Gardía H, et al. Tratamiento endovascular del aneurisma de aorta abdominal. Cir Gen. 2011;33:43-47.

15. Fink JG, Gutiérrez VS, Dena EE, Hurtado LLM, Zaldivar RFR. Tratamiento endovascular del aneurisma de aorta abdominal en pacientes mayores a 60 años: morbimortalidad perioperatoria. Cir Gen. 2002;24:34-39.

16. Ramírez-Vargas AF, Careaga-Reyna G, Téllez-Luna S, Argüero-Sánchez R. Tratamiento quirúrgico de los aneurismas de la aorta torácica. Rev Mex Cardiol. 2003;14:118-127.

17. Careaga.Reyna G, Ramírez-Vargas AF, Martínez Hernández-Magro R, Argüero-Sánchez R. Aneurisma de la aorta torácica en coartación aórtica sin cirugía previa. Presentación de dos casos. Cir Ciruj. 2009;77:61-63.

18. Galicia-Tornell MM, Marín-Solís B, Fuentes-Orozco C, Martínez-Martínez M, Villalpando-Mendoza E, Ramírez-Orozco F. Procedimiento de Bentall en la enfermedad aneurismática de la aorta ascendente: mortalidad hospitalaria. Cir Cir. 2010;78:45-51.

19. Soto-Hernández G, Alvarado-Lezama J, Bizueto-Rosas H, Madrid-Mier E, Martínez-Maya H, Mejía-Valero SA. Síndrome aortoduodenal secundario a un aneurisma de la aorta abdominal roto contenido. Rev Mex Angiol. 2014:42:95-101.

20. Morales-Victorino N, García-López S, Alcántara-Meléndez MA, Herrera-Gomar M, Guering-Eid L, González-Chon O. Reparación endovascular de aneurisma abdominal. Rev Invest Med Sur Mex. 2011;18:179-182.

21. Lacayo M, Lecuona N, Córdova P, Flores, S, Cárdenas, Á. Tratamiento endovascular de Aneurisma de Aorta abdominal en el Hospital General de México. Rev Med Hosp Gen Mex. 2016;79:161-164.

22. Candia-De la-Rosa RF, Córdova-González I, Rodríguez-Esqueda M Rico-Olvera H. Aneurisma infrarrenal de la aorta abdominal. Reporte de un caso. Rev Sanid Milit Mex. 2003:57:173-179.

23. Gutiérrez-Carreño AR, Lizola-Margolis RI, Mendieta-Hernández M. Aneurisma de aorta abdominal micótico roto. Manejo híbrido. Rev Mex Angiol. 2017:45:139-142.

24. Alcántara PA, Cogordan CJ, Reyes BC, Gómez CA, Huerta HD. Tratamiento no quirúrgico del aneurisma disecante de la aorta torácica mediante colocación de implante endovascular. Informe de un caso. Rev Sanid Milit Mex. 2000;54:214-216.

25. Mendoza-Álvarez SA, Fuentes-León J, Vargas-Ayala G, Hernández-González C, López-Arias G, Vera-Lastra O. Síndrome de Marfán, aneurisma toracoabdominal disecado y embarazo. Informe de un caso. Rev Med Inst Mex Seguro Soc. 2009;47:669-672.

26. Cohen-Mussali S, Zerrweck-López C, Rey-Rodríguez A, Valdés-Flores JA, Vidal-González P, Ramírez-Cerda C. Puente carótido-subclavio y prótesis endovascular como manejo híbrido de aneurisma de aorta torácica. An Med Mex. 2013;58:55-59.

27. Díaz-Quiroz G, Archundia-García A, Vázquez-Minero JC, Aceves-Chimal JL. Manejo quirúrgico de aneurisma de aorta ascendente. Presentación de un caso. Rev Esp Med Quir. 2003;8:52-55.

28. Martínez-Tapia AL, Robles-Pérez E, Jiménez-Hernández A, Ramos-Cano $\mathrm{VH}$. Aneurisma disecante de aorta ascendente. A propósito de un caso y revisión de la literatura. Rev Hosp Jua Mex. 2014;81:60-67.

29. Herrera-Gomar M, Alcántara-Meléndez MA, Lomelí-Estrada C, Mendoza-González C, Aguirre-Molina CA, Lorenzo-Negrete JA, et al. Aneurisma de aorta torácica por úlcera aterosclerótica penetrante: tratamiento endovascular, híbrido o cirugía. Revisión. Arch Cardiol Mex. 2009;79:140-146.

30. González-Álvarez CJ, Ordóñez-Toquero G, Rivera-Quintero E. Angioplastía con stent en el tratamiento de coartaciones aórticas críticas. Arch Cardiol Mex. 2006:76:424-430.

31. Uruchurtu E, Sánchez A, Pavía A, Hernández I, Valle L, Esquivel M Medel $\mathrm{O}$, Vargas $\mathrm{C}$. Aortoplastía en coartación aórtica congénita del adulto con balón y prótesis endovascular: resultados inmediatos y seguimiento a 6 meses. Arch Cardiol Mex. 2000;70:72-77.

32. Munayer-Calderón J, Zabal-Cerdeira C, Ledesma-Velazco M, Aldana-Pérez T, Ramírez-Reyes H, Lázaro-Castillo JL. Balloon angioplasty in aortic coarctation: a multicentric study in Mexico. Arch Cardiol Mex. 2002;72:20-28

33. Fink, G, Gutiérrez VS, Hurtado LLM. Cirugía endovascular mediante el implante de endoprótesis Talent en pacientes adultos con coartación de la aorta torácica: experiencia inicial de cuatro pacientes. Cir Gen. 2002;24:232-236.

34. Hernández-González M, Solorio S, Conde-Carmona I, Rangel-Abundis A, Ledesma M, Munayer J, et al. Intraluminal aortoplasty vs. surgical aortic resection in congenital aortic coarctation. A clinical random study in pediatric patients. Arch Med Res. 2003;34:305-310.
35. Márquéz-González H, De León-Mena S, Yáñez-Gutiérrez L, López-Gallegos D, Camargo-Zettina CO, Ortiz-Vázquez IC, et al. Late complications of aortic coarctation treatment: a Mexican cohort study with more than 10-year follow up. Rev Mex Cardiol. 2015;26:169-173.

36. Zabal C, Attie F, Rosas M, Buendía-Hernández A, García-Montes JA The adult patient with native coarctation of the aorta: balloon angioplasty or primary stenting? Heart. 2003;89:77-83.

37. Cervantes-Salazar JL, Ramírez-Marroquín S, Benita-Bordes A, Rosas-Peralta M, Attie F. Tratamiento quirúrgico de la coartación de aórtica. Resultados a largo plazo en el Instituto Nacional de Cardiología. Arch Cardiol Mex. 2006;76:63-68.

38. Buenfil-Medina JC Jiménez-Carbajal MG, Santiago-Herrara R, Fernández-Luna CP. Stent BeGraft Bentley ${ }^{\circledR}$ para tratamiento de coartación aórtica. Arch Cardiol Mex. 2018;88:313-315.

39. Maltos-Valdés W, Cáceres-Papadakis GU, Garza-Alatorre AG, Rodríguez-Balderrama I. Miocardiopatía dilatada secundaria a coartación de la aorta. Rev Mex Pediatr. 2004;71:25-27.

40. Rojas DMA, García MA. ¿Es la angioplastía el tratamiento de elección en la coartación de aorta? Presentación de un caso clínico y revisión de la literatura. Med Int Mex. 2010;25:524-529.

41. Rosas MA, Bolio A, Reyes NI, Sienra J. Coartación de la aorta y sibilancias. Bol Med Hosp Infant Mex. 1997:54:241-243.

42. Reyna-Cuevas VM, Segura-Vera L, Borbolla-Sala ME. Angioplastía con stent en coartación aórtica severa, con abordaje por arteria humeral. Salud Tab. 2013;19:66-70.

43. Parra-Bravo JR, Acosta-Valdez JL, Zepeda-Sanabria JR, Beirana-Palencia LG, Rodríguez-Hernández L, Estrada-Loza MJ, et al. Agenesia unilateral de una arteria pulmonar y coartación de aorta en un paciente con síndrome de Turner. Rev Mex Cardiol. 2002;13:162-170.

44. Guering, EL, Gaspar-Hernández J, González-Pacheco H, Acevedo-Gómez P, Ramírez-Marroquín S, Herrera-Alarcón V, et al. Complicated acute aortic syndromes affecting the descending thoracic aorta: endovascular treatment compared with open repair. Clin Cardiol. 2015:38:585-589.

45. Guering, EL, Gaspar, J, Meléndez-Ramírez, G, Cervantes, J, González-Pacheco, H., Dámas de los Santos, F, et al. Endovascular treatment of type B dissection in patients with Marfan syndrome: mid-term outcomes and aortic remodeling. Cathether Cardiovasc Interv. 2013; 82:E898-E905.

46. Hinojosa CA, Anaya-Ayala JE, Torres-Machorro A, Lizola R, Laparra-Escareno $\mathrm{H}$. Middle aortic syndrome in Takayasu's arteritis: report of two surgical cases. Ann Vasc Surg. 2015;34:e13-e17.

47. Mijangos-Vázquez R, Miranda-Chávez IO, Soto-López ME, Patiño-Bahena EJ, Cano-Zárate R, Covarrubias-Flores AC, et al. Anomalous origin of pulmonary branches from the ascending aorta. A report of five cases and review of the literature. J Cardiol Cases. 2015;11:1-6.

48. Herrera-Gómez A, Ortega-Gutiérrez C, Luna-Ortiz K, De-León-Zaragoza L. Resección arterial de grandes vasos debido a tumor germinal residual retroperitoneal. Tres casos. Cir Gen. 2011;33:127-131.

49. Fink JG, Fernández SE, Gutiérrez VS, Hurtado LLM, Ruiz DA. Manejo endovascular de la transección de aorta torácica por trauma cerrado de aorta: informe de dos pacientes. Cir Gen. 2002;24:144-147.

50. Sigler-Morales L, Martínez-López C, González-Valenzuela J, Guevara-Saldívar MI, Jaramillo-Talavera J. Fístula aortocava y embolia por proyectil de arma de fuego a la arteria poplítea. Cir Cir. 1997;65:56-58.

51. Delgadillo GH, Velásquez JA, Fening J, Campos F, Cervantes J, Medina B. Fístula aorto-gástrica. Informe de un caso tratado con reparación de la aorta, gastrectomía total y esófago-yeyuno-anastomosis en $Y$ de Roux. Cir Gen. 2003;25:234-237.

52. Rocha-Rodríguez MG, Rodríguez-Blas Al, Balcázar-Vázquez R, Casian-Castellanos GA, Flores-Cabos AE. Fístula aorto cava asociada con aneurisma de aorta abdominal: reporte de un caso. Rev Hosp Jua Mex. 2010;77:133-137.

53. Vidal-González $P$, Mussan-Chelminsky G, Kunz-Martínez W, Jonguitud-Bulos V, Rey-Rodríguez A, Cohen-Mussali S. Tratamiento endoluminal para ruptura aórtica por trauma. Reporte de un caso y revisión de la literatura. Cir Cir. 2007;75:297-302.

54. Gamboa R, Ríos-Méndez RE, Solernó R, Giachello F Videla-Lynch A, Sarmiento RA. Exclusión percutánea de seudoaneurisma aórtico abdominal traumático desde acceso braquial. Arch Cardiol Mex. 2011; 82:34-36.

55. López RJA, Parada LJR, Sagahón MJA, Corona PMA, Aguilar AE, Gómez $\mathrm{ML}$, et al. Reparación quirúrgica de doble arco aórtico. Rev Esp Med Quir. 2012;17:210-213. 